\title{
Relaciones entre el autoconcepto relacional, la elección de metas y la satisfacción de necesidades psicológicas en estudiantes universitarios*
}

\author{
Relations between the Relational Self-Construal, the Choice of \\ Goals and Psychological Need Satisfaction of University Students
}

Recibido: marzo 25 de 2013 | Revisado: enero 24 de 2014 | Aceptado: abril 15 de 2014

\author{
ElENA GÁMEz ARMas** \\ Jose Miguel Díaz Gómez \\ Hipólito MARrero HernáNDEZ \\ Universidad de La Laguna, Tenerife, España \\ M az Galindo Galindo \\ Alicia Breva Asensio *** \\ Universidad de Sevilla, España
}

doi.org/10.11144/Javeriana.UPSY13-4.rarm

Para citar este artículo: Gámez, E., Díaz, J. M., Marrero, H., Galindo, M. P., \& Breva, A. (2014). Relaciones entre el autoconcepto relacional, la elección de metas y la satisfacción de necesidades psicológicas en estudiantes universitarios. Universitas Psychologica, 13(4), 1289-1303. http://dx.doi. org/10.11144/Javeriana.UPSY13-4.rarm

* Este trabajo se ha realizado dentro del proyecto PSI2008-00015/PSIC financiado por el Ministerio de Ciencia e Innovación del gobierno de España.

** Facultad Psicología. Campus de Guajara s/n, 38071. La Laguna. S/C de Tenerife, España. Correos electrónicos: egamez@ull.es; jmdiaz@ull.es; hmarrero@ull.es; mpaz@ull.es

**** Facultad Psicología. Camilo José Cela s/n, 41018, Sevilla, España. Correo electrónico: alicia@us.es

\section{RESUMEN}

El objetivo de este trabajo fue estudiar cómo ciertas características del autoconcepto pueden influir en la elección de unas u otras metas en estudiantes universitarios. Se utilizó una adaptación al castellano de la escala Relational-Interdependent Self-Construal ([RISC]; Cross, Bacon, \& Morris, 2000) para medir el grado en el que los estudiantes incorporan sus relaciones interpersonales significativas en la construcción del autoconcepto. En una muestra de 389 universitarios españoles (rango de edad 16-47, $M=19.28$; $D E=3.15)$ se efectuaron distintos contrastes que mostraron diferencias significativas asociadas al género $(\varnothing=59 \%$ y $\hat{\sigma}=41 \%$ con tamaños de efecto $d 0.25-0.55$ ) y a las puntuaciones (altas frente a bajas, $d 0.42-0.91$ ) en la escala del RISC. Asimismo, esta variable de diferencias individuales apareció como predictora significativa en la elección de determinado tipo de metas y de la satisfacción de las necesidades psicológicas de autonomía, competencia y vinculación. Estos resultados tienen implicaciones en la elección de estudios y en lo que los estudiantes esperan encontrar en la universidad. Palabras clave autoconcepto relacional; metas interpersonales; necesidades psicológicas; diferencias individuales

\section{A B S T R A C T}

The aim of this study was to explore the role of specific features of the selfconcept that could be associated with the choice of life goals. We developed an adaptation of the RISC (Relational-Interdependent Self-Construal; Cross, Bacon, \& Morris 2000) to directly assess the degree to which individuals define themselves in terms of close relationships. The scale was translated into Spanish and its psychometric properties were analyzed with data from a sample of 389 university students (age range 16-47, $M=19.28$, $\mathrm{ST}=3.15)$. A set of contrast showed significant differences between men and women $(+=59 \%$ y $\hat{\jmath}=41 \%$ effect size d $0.25-0.55)$. Significant differences appeared also regarding extrinsic and intrinsic goals, interpersonal goals and needs satisfaction when students with a highly relational self-construal were compared with students with a low relational one (d 0.42-0.91). These 
results have implications for the choice of studies and what students expect in university life.

Keywords

relational self; interpersonal goals; psychological needs; individual differences

Uno de los temas mejor estudiados en la Teoría de la Autodeterminación (Deci \& Ryan, 2000) son las consecuencias motivacionales de tener unas metas extrínsecas o intrínsecas. Las primeras, entre las que se encontrarían el éxito financiero o el atractivo físico, están relacionadas con la obtención de algún tipo de refuerzo explícito o reconocimiento social. En contraposición, las metas intrínsecas producen satisfacción en sí mismas, sin esperar nada a cambio. Son un fin y no, como las extrínsecas, un medio para obtener un refuerzo. Metas intrínsecas son la autoaceptación, la afiliación o el sentirse parte de una comunidad (Grouzet et al., 2005; Kasser, 2002; Kasser \& Ryan, 1996; Sheldon, Gunz, Nichols, \& Ferguson, 2010). Las personas adoptan metas de una y otra naturaleza, diferenciándose en la importancia que conceden y el apego que tienen a cada una de ellas. Aunque las personas se diferencien en la constelación de metas personales e impersonales, también se parecen mucho en los tipos de meta que persiguen. En un estudio transcultural desarrollado en 15 naciones, Grouzet et al. (2005) encontraron que existían 11 tipos de metas que se podían organizar en torno a dos dimensiones ortogonales. La primera diferenciaría lo extrínseco frente a lo intrínseco; la segunda, la autotrascendencia (expresada en metas de naturaleza espiritual) frente a metas ancladas al mundo físico, como la seguridad o la salud. Aunque existían pequeñas diferencias entre algunos países, las dos dimensiones mostraron buenos índices de fiabilidad en el conjunto de las naciones estudiadas, independientemente de que estas fueran más o menos ricas, según el índice del PIB per capita.

Aunque existan diferencias entre las personas y entre las culturas en la importancia que se atribuya a la consecución de un tipo y otro de meta, lo que sí parece un hecho bien constatado es que las personas que se inclinan predominantemente por las metas extrínsecas se sienten más infelices y con un menor bienestar subjetivo, en comparación con las personas que se orientan a las metas intrínsecas (Sheldon 2005; Sheldon, Ryan, Deci, \& Kaisser, 2004; Vansteenkiste, Soenes, \& Duriez, 2008).

Para contestar a la pregunta de por qué las personas eligen un tipo de meta u otro, se necesitaría un espacio mayor que el de este artículo. Habría que mencionar los múltiples factores que intervienen en las elecciones de cada individuo. Por un lado, factores sociales y culturales que incitan y animan a preferir unas metas más que otras (en el caso de la cultura occidental, los mass-media, por ejemplo). Por otra parte, hay que tener en cuenta los factores personales que recorren la historia de cada individuo (Kasser, 2002), como la inseguridad emocional (Sheldon \& Kasser, 2008), la construcción del autoconcepto (Cross, Hardin, \& Gercek-Swing, 2009), la motivación aproximativa o evitativa (Carver, 2006), la satisfacción de necesidades (Deci \& Ryan, 2002), etc.

El trabajo que se presenta aquí se centra en el papel que pueden desempeñar ciertas características del autoconcepto en la elección de unas metas u otras, en los estudiantes universitarios. En concreto, en lo que Susan Cross (2000, p.795) denomina "la construcción del autoconconcepto dependiente de las relaciones" (the relational-interdependent self-construal), esto es, en qué medida las personas incorporan las relaciones interpersonales significativas en su definición del self.

La importancia dada a esta dimensión del autoconcepto procede de la psicología cultural que emergió en la década de los 90 del pasado siglo, centrada en estudiar la influencia de las llamadas culturas individualistas frente a las colectivistas en la conducta social y en el pensamiento (Kitayama, 2002; Nisbett, 2003; Triandis, 1994). Desde esta perspectiva, en los últimos veinte años no se ha dejado de investigar el qué, el cómo y el porqué del proceso de construcción del autoconcepto (self construal) teniendo en cuenta esa relación indi- 
visible entre mente y cultura (Cross, Hardin, \& Gercek-Swing, 2011).

En un artículo paradigmático, Markus y Kitayama (1991) acuñan el término self construal ${ }^{1}$, para describir la forma en la que los norteamericanos y japoneses definen e interpretan su autoconcepto (self). Estos autores identifican dos procesos claramente diferenciados: uno independiente y otro interdependiente. En el primero, característico de europeos y americanos, el autoconcepto se construye como algo individual, separado de los otros. Como dicen Cross et al. (2011) a la pregunta clave de "iquién soy yo?" se responde haciendo referencia a rasgos estables del tipo: "soy inteligente", "tímido", etc., o en que se diferencian del resto de las personas, por ejemplo, ser especialmente creativo o más hipocondriaco que la mayoría. Desde esta interpretación del yo, la estabilidad de estas características en diferentes contextos y a lo largo del tiempo es una señal de madurez. Ser alguien que se distingue de los otros es también una señal que contribuye a mejorar la autoestima. Las relaciones interpersonales son importantes, pero desde la perspectiva de cómo benefician a la individualidad (por ejemplo: validando la autoestima) o como fuente de comparación social ("soy más creativo porque me comparo con mis amigos"). En contraste, el proceso de autoconstrucción interdependiente, característico de los japoneses y otras culturas asiáticas y latinoamericanas, se define en conexión con los otros y con las relaciones que se mantienen con ellos. La respuesta a "iquién soy?" se caracteriza por la referencia a las relaciones significativas (del tipo "soy madre", "amigo", "colega") o de pertenencia a un grupo ("soy latino", "soy Kawasaki”, etc.). La autoestima mejora a partir de las habilidades relacionadas con el ajuste en el grupo, y la madurez se mide por el autocontrol emocional o la capacidad de responder a diferentes demandas situacionales que tengan como objetivo la armonía del grupo.

Según Markus y Kitayama (1991) cada persona incorpora en su autoconcepto la dimensión independiente e interdependiente y es el contexto cultural

1 Se podría traducir por interpretación del yo, construcción del autoconcepto o del sí mismo. el que poco a poco estimula el desarrollo de una de estas dimensiones en detrimento de la otra. Así, los Estados Unidos de América y Japón representarían dos culturas prototípicas que promueven uno u otro proceso en la construcción del autoconcepto.

Existen varias escalas para medir las dos dimensiones propuestas por Markus y Kitayama (1991) de las cuales las más utilizadas han sido las SelfConstrual Scales ([SCS]; Singelis, 1994). En su versión original la escala permite medir las dos dimensiones que teóricamente son ortogonales, obteniéndose dos puntuaciones que confirman las diferencias esperadas entre culturas orientales y occidentales (Singelis, 1994; Singelis \& Brown, 1995; Singelis, Bond, Lai, \& Sarkey, 1999). Con el paso de los años, otros autores han añadido más ítems a la escala original e incluso han ampliado el número de factores que explican estas dos dimensiones del autoconcepto, proponiendo una estructura multidimensional que se ajusta mejor a los datos de estas nuevas escalas, con cuatro factores para la dimensión independiente (asertividad o autonomía, individualismo, consistencia y primacía del self) y dos factores para la interdependiente (estima del grupo e interdependencia relacional) (Hardin, 2006).

En el contexto del desarrollo de estas nuevas medidas es donde aparece en el 2000 la escala de Cross y su colaboradores (Relational-Interdependient Self-Construal Scale [RISC]) para medir el aspecto relacional de la dimensión interdependiente. La escala RISC presenta índices buenos de fiabilidad (entre 0.77 y 0.9) así como de estabilidad test-retest (Cross et al., 2000). En la mayoría de los estudios las mujeres puntúan más alto que los hombres (diferencias entre 0.17 y 0.57). Las puntuaciones en la escala correlacionan positiva y moderadamente con otras medidas de la dimensión relacional pero con ninguna medida de independencia (Cross et al., 2009). La escala tiene una estructura unidimensional de tal forma que funciona como una variable de diferencias individuales con la que se pueden formar grupos de altos y bajos en la dimensión relacionalinterdependiente. Del conjunto de trabajos en los que se ha comparado estos dos grupos se sabe que las personas que puntúan alto en el RISC tienen en cuenta las opiniones y necesidades de los otros en 
los procesos de toma de decisiones, son más abiertas y responden más fácilmente a las otras personas en la primera interacción con ellas (Cross et al., 2000), tienen una mayor atención selectiva, una red cognitiva más elaborada sobre sus relaciones y un mejor recuerdo de la información relacional (Cross, Morris, \& Gore, 2002) y están más interesados en buscar metas que tengan en cuenta sus relaciones cercanas (Gore, Cross, \& Kanagawa, 2009).

Uno de los objetivos específicos de este trabajo fue estudiar con más de detalle las propiedades psicométricas de esta escala y su adecuación para utilizarla en castellano. Un segundo objetivo, relacionado con validar el uso de dicha escala, fue comprobar algunas de las predicciones que se han constatado en muestras anglosajonas, cuando se compara a personas con un alto "autoconcepto-relacional" frente a aquellas que presentan un bajo autoconcepto-relacional. Un tercer objetivo consistía en comprobar la influencia de esta medida de diferencias individuales en las metas de los estudiantes y en la satisfacción de sus necesidades psicológicas.

Conocer el perfil de los estudiantes universitarios en términos de su representación de los otros, puede tener algunas aplicaciones relevantes. Por un lado, puede ser informativo de la adecuación de la elección de los estudiantes cuando piensan en cursar estudios que tienen que ver con profesiones con vocación de servicio tales como psicología, enfermería, medicina, trabajo social, etc. Por otro lado, estudios recientes demuestran que los estudiantes universitarios que tienen en cuenta a los otros despliegan más conductas de ayuda, de colaboración con los otros y de participación en la vida universitaria (Mattingly, Oswald, \& Clark, 2011), al mismo tiempo que son más compasivos consigo mismos y con los otros (Breines \& Chen, 2013).

A partir de los trabajos de Cross et al., se pueden tener algunas expectativas. En primer lugar, se esperaba constatar las diferencias de género señaladas más arriba, esto es, puntuaciones significativamente más altas de las mujeres en el RISC que los hombres. En segundo lugar, las diferencias entre estudiantes con

2 Se podría traducir por yo-relacional en lugar de autoconceptorelacional. puntuaciones altas y bajas en el RISC en la importancia y probabilidad concedida a las metas de naturaleza interpersonal, pues serían las que se relacionan directamente con su sensibilidad para estos temas. Por último, si las predicciones anteriores se confirmaran y la variable RISC apareciera como una variable de diferencias individuales bien definida, tendría que mostrar su capacidad para predecir la elección de metas relacionadas con los otros y la satisfacción de las necesidades psicológicas de autonomía, competencia y vinculación, tal como las define la Teoría de la Autodeterminación (Deci \& Ryan, 2000).

\section{Método}

\section{Participantes}

Los participantes fueron 605 estudiantes de universidad pública. El 59\% eran mujeres y el 41\%, hombres, de edades comprendidas entre los 16 y los 47 años $(M=19.28 ; D E=3.15)$. La muestra se obtuvo de personas matriculadas en el primer curso de la licenciatura y el grado de Psicología de tres cursos, 2006-2007 (30\%), 2007-2008 (36\%) y 2010-2011 (34\%). Del conjunto inicial, una submuestra de 389 sujetos completó el conjunto de escalas que ha dado lugar a los distintos análisis que se expondrán a continuación. El resto, 216 participantes, fueron destinados exclusivamente al análisis factorial confirmatorio, al que se hará referencia más adelante.

El procedimiento para el manejo de casos perdidos consistió en asignar a los valores perdidos la media del sujeto en el resto de los ítems de la escala con valores válidos. El conjunto de valores perdidos no llegó al 5\% del conjunto total. Todos los estudiantes participaron de forma voluntaria para obtener créditos extra en una asignatura.

\section{Instrumentos}

Escala de Interdependencia Relacional ([RISC]; Relational-Interdependient SelfConstrual Scale; Cross et al., 2000)

Mide la tendencia de cada persona a pensar en sí misma en términos de las relaciones cercanas con 
los otros. Los participantes tienen que valorar en una escala Likert del 1 al 7 once frases del tipo "Mis relaciones son un importante reflejo de quién soy". Quienes puntúan alto dan mucha importancia a sus relaciones cercanas, se comprometen con ellas y, cuando toman decisiones, tienen en cuenta las necesidades y los deseos de los otros. En palabras de sus autores, son personas que "incluyen a los otros en el self”. Siguiendo las recomendaciones de los especialistas en adaptaciones de los test (Muñiz, \& Hambleton, 1996) se realizó una traducción inversa de la escala original en inglés, corrigiendo las expresiones que se consideró podían resultar extrañas para los estudiantes universitarios.

\section{Cuestionario MOPI (Gámez $\mathcal{G}$}

Marrero, 2001, 2003)

Este cuestionario se utilizó para estudiar los Motivos Interpersonales de los estudiantes del primer curso de universidad. Lo forman 45 ítems tipo Likert, con una escala del 1 al 7. Cada ítem expresa una afirmación que completa la frase "Has elegido esta carrera porque...." El conjunto permite rastrear aspectos que tienen que ver con situaciones sociales e interpersonales, tales como "(...) quieres emprender un camino en la vida y llegar al final"; o "(...) siempre has tenido deseos de ayudar a los demás”. Para la muestra de este estudio, y tal como hicieron los autores de la escala, se realizó un AF de componentes principales con rotación VARIMAX, que arrojó un modelo de 6 factores con valores propios iguales o superiores a 1.5 que explicaban el $46 \%$ de la varianza total $(\mathrm{KMO}=0.89$ y B $=6232.64, p$ $<0.001)$. Se trata de un modelo donde las metas se agrupan en seis categorías: superación de problemas personales ( $\alpha=0.8)$, logro $(\alpha=0.81)$, relaciones interpersonales $(\alpha=0.77)$, poder $(\alpha=0.7)$, afiliación $(\alpha=0.66)$ y conocimiento $(\alpha=0.67)$.

\section{Cuestionario de Nivel de Aspiración}

(Grouzet et al., 2005)

El cuestionario se emplea para evaluar las Metas Vitales de los estudiantes. En este estudio se utilizó para conocer las metas de los estudiantes en once dominios diferentes. Para ello, se les preguntaba por la importancia y la probabilidad de elegir un conjunto de 57 metas futuras: por ejemplo, "Que siempre exista alguien cerca de mí para cuidarme" o "Ser eficiente". Los autores agrupan las metas en cuatro cuadrantes que resultan de cruzar la dimensión intrínseco-extrínseco y la dimensión yo trascendente y yo físico. Así, en el cuadrante intrínseco-yo trascendente las metas serían espiritualidad, pertenecer a una comunidad, afiliación y autoaceptación. Las metas del cuadrante extrínseco-yo trascendente serían conformidad, popularidad e imagen. Las metas del cuadrante intrínseco-yo físico, salud física, seguridad y hedonismo Y por último, en el cuadrante extrínseco-yo físico se situarían la meta de obtener dinero. Esta escala se validó en el estudio arriba citado, realizado en quince países entre los que se encontraba España. Para la muestra de este trabajo los índices de fiabilidad fueron: para las metas extrínsecas, que incluían dinero, imagen y popularidad, descartando conformidad $(\alpha \leq$ $0.5), \alpha=0.81$ para la importancia y $\alpha=0.8$ para la probabilidad; en el caso de las metas intrínsecas que incluían autoaceptación, afiliación, comunidad y salud física, descartando seguridad $(\alpha \leq 0.55)$, $\alpha=0.75$ para la importancia y $\alpha=0.77$, para la probabilidad. En el caso de las metas relacionadas con la dimensión del mundo físico incluían hedonismo, salud y dinero, descartando seguridad $(\alpha \leq$ $0.5), \alpha=0.67$ para la importancia y $\alpha=0.71$, para la probabilidad. La dimensión del yo-trascendente que incluye las metas de conformidad, comunidad y espiritualidad fue rechaza porque las $\alpha<0.5$, tanto para la evaluación de la importancia como para la probabilidad de conseguirlas.

\section{Escala de Necesidades Psicológicas Básicas ([BANES]; Basic Need Satisfaction in General; Deci Eु Ryan, 2000, 2002)}

Mide el grado en que se experimenta satisfacción con cada una de las necesidades psicológicas organísmicas definidas en la Teoría de la Autodeterminación (Deci \& Ryan, 2000). Es decir, en qué medida las personas sienten satisfechas las tres necesidades básicas de autonomía, competencia y 
vinculación. Los participantes tienen que valorar en una escala Likert del 1 al 7 frases del tipo "Me siento libre de decidir cómo quiero vivir mi vida" o "Me siento presionado". Los índices de fiabilidad fueron mínimamente aceptables para las tres necesidades: autonomía $(\alpha=0.62)$, competencia $(\alpha=$ $0.64)$ y vinculación $(\alpha=0.67)$.

Positive and Negative Affect Schedule ([PANAS]; Watson, Clark, \& Tellegen, 1988). Se utilizó la adaptación al español de la escala PANAS diseñada para evaluar las emociones positivas y negativas que sienten las personas en su vida cotidiana. La escala incluye 10 ítems tipo Likert para evaluar las emociones positivas PA (en qué medida una persona se siente activa, entusiasta y alerta) y 10 ítems para evaluar las emociones negativas NA (en qué medida la persona se siente culpable, enfadada o triste). Los participantes rellenaron el SPANAS evaluando sus emociones y sentimientos habituales. Los índices de fiabilidad para la muestra fueron: PA $(\alpha=0.84)$ y NA $(\alpha=0.85)$.

\section{Procedimiento y análisis}

Los participantes fueron convocados a una hora cómoda para ellos, en un aula, donde recibían un cuadernillo con el conjunto de las escalas. Antes de comenzar a rellenar los cuadernillos se les aseguró la confidencialidad de sus respuestas y la importancia de su sinceridad. Previamente, se les había informado de su carácter voluntario, por lo que se asumía que su participación en la investigación equivalía a otorgar el consentimiento para ello. Tras ser revisados, se rechazaron los protocolos que presentaban alguna de las escalas sin contestar. Los datos se introdujeron en una hoja del programa SPSS 17.0 para Windows con el que se realizaron los análisis estadísticos, a excepción de los análisis factoriales confirmatorios, para lo cual se utilizó la ampliación AMOS 18.0.

La planificación de los análisis fue la siguiente: en primer lugar, la realización de los análisis factoriales exploratorio y confirmatorio, con dos muestras diferentes, para comprobar la unidimensionalidad del constructo RISC y los ítems que constituirían nuestra medida, para los análisis posteriores.
Una vez obtenida la medida de la escala RISC en español, RISC-e, se llevaron a cabo análisis correlaciónales con el resto de las escalas y el análisis de los estadísticos descriptivos de las variables utilizadas. A continuación, se elaboraron contrastes de medias entre los grupos de hombres y mujeres y de estudiantes altos y bajos en el RISC-e con respecto al resto de las variables. Para estos contrastes de medias se utilizó la $t$ de Student y se calculó la diferencia de medias tipificada, $d$, con vistas a conocer el tamaño del efecto de dichas diferencias. Seguidamente, se realizaron ANOVAS de dos factores, con el objeto de conocer la interacción entre la variable género y las puntuaciones en la Escala RISC-e sobre la importancia concedida a las metas y a las necesidades psicológicas. Por último, se hicieron varios análisis de regresión para explorar el carácter predictivo de RISC-e y las metas intrínsecas, en la satisfacción de las necesidades psicológicas de autonomía, competencia y vinculación.

\section{Resultados}

\section{Propiedades psicométricas de la} Escala de Interdependencia Relacional traducida al castellano (RISC-e)

\section{Análisis factorial exploratorio}

En primer lugar se hizo un análisis factorial exploratorio con la submuestra de 389 participantes. Los valores correspondientes a la medida de adecuación muestral de Kaiser-Meyer-Olkin (KMO) y la prueba de esfericidad de Bartlett fueron aceptables $\chi_{(55)}^{2}=$ 920.249; $p=0.0001 ; \mathrm{KMO}=0.799)$. La factorización se llevó a cabo por el método de extracción de componentes principales (el mismo utilizado por Cross et al., 2000). Aparecieron dos factores con valores propios mayores que 1 . El primer factor reúne 9 ítems cuya carga factorial va de 0.52 a 0.71 . El segundo factor incluye los dos ítems negativos de la escala (ítems 8 y 9), cuyos pesos factoriales son 0.78 y 0.83 . El primer factor explica el $32 \%$ de la varianza y el segundo el 14\%.

Si se eliminan los ítems 8 y 9 y se hace un nuevo análisis factorial, aumentan el índice $\mathrm{KMO}=0.829$ 
y la prueba Bartlett $\left(\chi_{(36)}^{2}=796.221 ; p=0.0001\right)$. La nueva factorización arroja un solo factor que explica el 39\% de la varianza con los 9 ítems cuya carga factorial va de 0.52 a 0.72 . El valor alfa de Cronbach con los 11 ítems originales fue de 0.77 y de 0.8 , al eliminar los ítems 8 y 9.

\section{Análisis factorial confirmatorio}

Con el fin de evaluar la estructura factorial de la escala eliminando los ítems 8 y 9, se realizó un análisis factorial confirmatorio utilizando el paquete estadístico AMOS 18.0. Para este análisis, se acudió al resto de los participantes, es decir, una submuestra de 216 estudiantes, diferentes a los utilizados en el análisis anterior. El 80\% eran mujeres y sus edades estaban comprendidas entre los 17 y los 34 años.

En el análisis de la submuestra, el coeficiente Mardia fue más alto de lo deseable (curtosis multivariado = 9.7). Según los especialista en el AFC (Byrne, 2010), el método de máxima verosimilitud, el más utilizado, puede ser problemático, por lo que una alternativa para unos datos que no cumplen con los criterios de normalidad es el método ADF, distribución libre asintótica. Para utilizar este método, el tamaño de la muestra debe ser, como mínimo, 10 veces mayor que el número de parámetros estimados. En el presente caso, el modelo cuenta con 21 parámetros por lo que el tamaño de la muestra permite aplicar este método. Para aceptar o rechazar un modelo, se emplea una combinación de varios índices. $\chi^{2}$ debería tener un valor no significativo $(p>0.05)$, pero es muy sensible al tamaño de la muestra (Jöreskog \& Sörbom, 1993) por lo que se suele calcular el $\chi^{2} / g l$, que se considera aceptable cuando es $<5$ (Bentler, 1989). En este caso, los valores fueron $\chi_{(24, \mathrm{~N}=216)}^{2}=26.346 ; p=0.336 \mathrm{y}$ $\chi^{2} / g l=1.1$. Los índices CFI, IFI y TLI se basan en la comparación entre el modelo hipotetizado y el modelo nulo, considerándose aceptables los valores superiores a 0.9. Los valores fueron bastante buenos $\mathrm{CFI}=0.98, \mathrm{IFI}=0.98$ y TLI $=0.97$. Por último el RMSEA (Root Mean Square Error of Aproximation) con valores $<0.05$ se considera un indicador de un buen modelo. En el caso del estudio RMSEA $=0.021$. Los pesos de regresión estandarizados fue- ron todos significativos y oscilaron entre 0.4 y 0.7 . El valor alfa de Cronbach para esta submuestra con los 9 ítems fue de 0.83 .

Análisis de la escala RISC-e con el resto de las variables del estudio

Con los resultados obtenidos de los análisis factoriales y la consistencia interna se consideró apropiado eliminar los ítems 8 y 9 de todos los análisis posteriores. La escala traducida al español y con dos ítems menos que la original (ver en el Apéndice) ahora se denominará RISC-e.

\section{Estadísticos descriptivos y correlaciones}

En la Tabla 1, se muestran los estadísticos descripticos, medias, desviaciones estándar y correlaciones entre las variables utilizadas.

\section{Diferencias de género}

En la Tabla 2, aparecen las diferencias de medias encontradas en las variables del estudio, en función del género de los sujetos. Tal como se esperaba, la prueba $t$ para muestras independientes reveló que las mujeres puntúan más alto que los hombres en la escala del RISC-e $\left(M_{\text {mujeres }}=4.82\right.$ y $M_{\text {hombres }}=$ 4.61), aunque el tamaño de la diferencia no es muy grande $(d=0.25)$. Esta diferencia ha sido ampliamente constatada en las investigaciones previas de Cross y sus colaboradores $(2000,2002,2011,2009)$. También resultaron diferencias significativas entre hombres y mujeres en las metas intrínsecas, tanto en la importancia como en la probabilidad de conseguirlas. Como se ve en la Tabla 2, en ambos casos son las mujeres las que puntuaron más alto. Por el contrario, en el caso de las emociones, evaluadas con el SPANAS, son los hombres los que puntuaron más alto, tanto para las emociones positivas como para las negativas.

\section{Diferencias entre altos y bajos en el RISC-e}

Del conjunto de la muestra se seleccionaron 119 estudiantes en dos grupos extremos que represen- 
Elena Gámez Armas, Jose Miguel díaz Gómez, Hipólito Marrero Hernández, Ma Paz Galindo Galindo, Alicia Breva Asensio

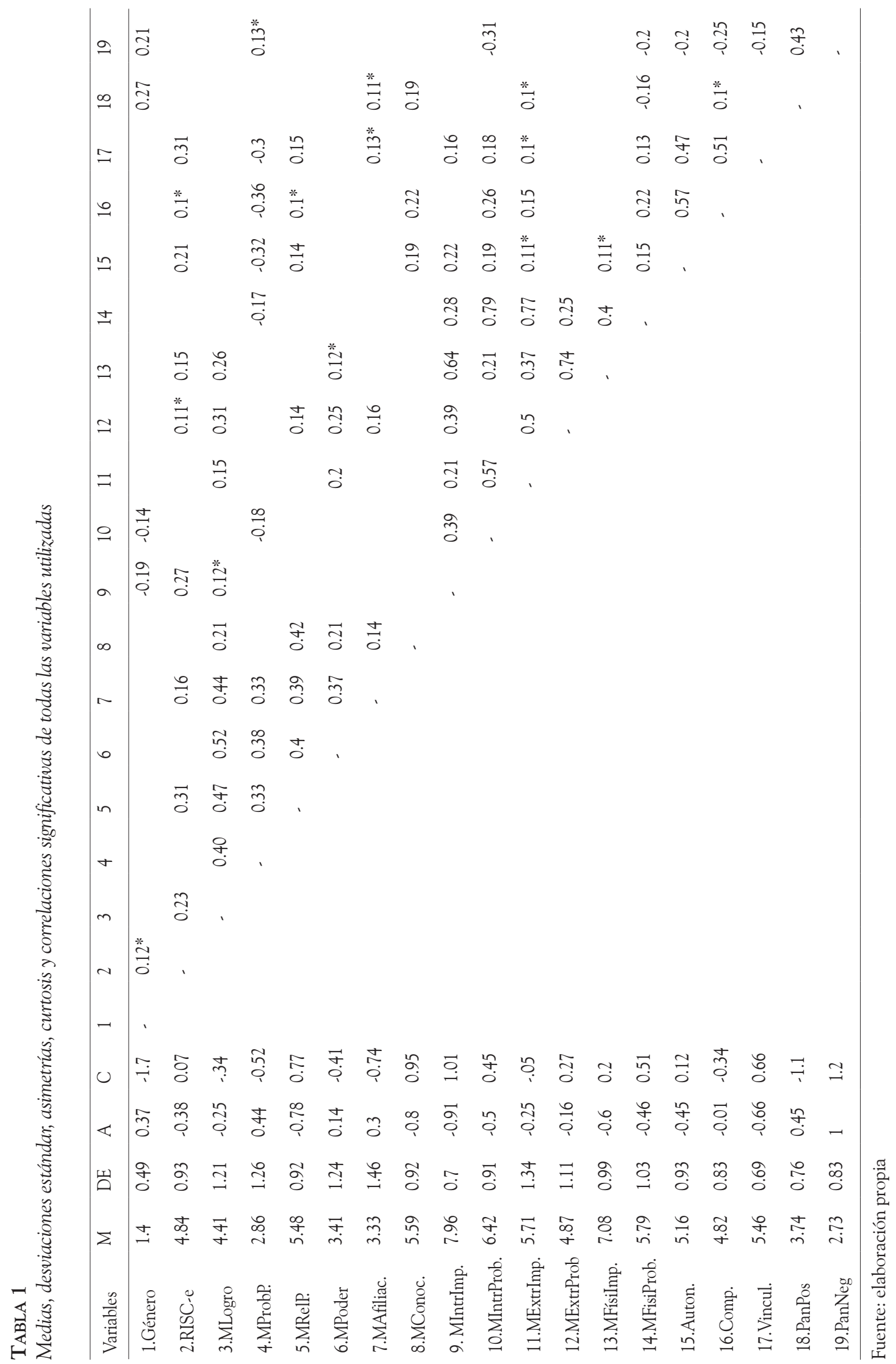


RELACIONES ENTRE EL AUTOCONCEPTO RELACIONAL, LA ELECCIÓN DE METAS Y LA

SATISFACCIÓN DE NECESIDADES PSICOLÓGICAS EN ESTUDIANTES UNIVERSITARIOS

TABLA 2

Medias y desviaciones estándar (entre paréntesis) de hombres y mujeres en distintas variables del estudio

\begin{tabular}{lccccc}
\hline \multicolumn{1}{c}{ Variable } & $\begin{array}{c}\text { Mujeres } \\
(\mathrm{N}=230)\end{array}$ & $\begin{array}{c}\text { Hombre } \\
(\mathrm{N}=159)\end{array}$ & $\mathrm{t}$ & $\mathrm{P}$ & $\mathrm{d}$ \\
\hline Risc-e & $4.8(0.8)$ & $4.6(0.9)$ & 2.55 & 0.01 & 0.25 \\
Metas Intrínsecas I & $8.1(0.7)$ & $7.8(0.7)$ & 3.8 & 0.001 & 0.38 \\
Metas Intrínsecas P & $6.5(0.9)$ & $6.2(0.9)$ & 2.7 & 0.01 & 0.27 \\
Emociones Positivas & $3.6(0.7)$ & $4.0(0.8)$ & 5.33 & 0.001 & 0.55 \\
Emociones Negativas & $2.6(0.9)$ & $2.9(0.7)$ & 3.93 & 0.001 & 0.41 \\
\hline
\end{tabular}

Fuente: elaboración propia

taban a los estudiantes con altas y bajas puntuaciones en el RISC-e. El grupo con bajas puntuaciones incluía a 62 estudiantes hasta el percentil 16 con valores $\leq 3.89$ y el grupo con altas puntuaciones incluía a 57 estudiantes a partir del percentil 85 con valores $\geq 5.8$. Aunque normalmente se utilizan los percentiles 25 y 75 para estas comparaciones, la media de las puntuaciones en la escala RISC-e es bastante alta $\mathrm{M}=4.8$, la puntuación que se corresponde con el percentil 25 es 4.18 y con el 75, 5.27. Por esta razón, se modificaron un poco esos percentiles con el objetivo de conseguir grupos extremos (altos frente a bajos) claramente diferenciados.

La prueba t para muestras independientes reveló que los estudiantes altos en el RISC-e con respecto a los bajos daban más importancia a las metas de logro, de relaciones personales, de afiliación, intrínsecas y metas relacionadas con el mundo físico. También percibían una mayor satisfacción en las necesidades psicológicas de autonomía y vinculación y una mayor activación de la motivación aproximativa. En la Tabla 3 se presentan las me- dias, desviaciones, valores de las $t$ para cada grupo y diferencia media tipificada.

Interacciones entre género y altos y bajos en el RISC-e

Teniendo en cuenta el peso de la variable género en las medidas del self se pretendió determinar las relaciones de dicha variable con los grupos altos y bajos en el RISC-e. La intención era explorar el papel modulador de la variable RISC-e en la importancia concedida a unas y otras metas y en la satisfacción de las necesidades psicológicas. Para esto, se efectuó el análisis de varianza de dos factores de efectos fijos, cuyos resultados revelaron una interacción significativa para las metas de afiliación, $F_{(1,115)}=5.84, p<0.02, \eta_{p}^{2}=0.05$, de superación de problemas personales, $F_{(1,115)}=4.59, p<0.05$, $\eta_{\mathrm{p}}^{2}=0.04$ y para la necesidad de vinculación, $F_{(1,115)}$ $=6.7, p<0.05, \eta_{p}^{2}=0.06$.

En el primer caso, como se muestra en la Figura 1 , son los estudiantes los que modulan su interés por las metas de afiliación en función de su puntuación

TABLA 3

Medias y desviaciones estándar (entre paréntesis) de altos y bajos en el RISC-e en distintas variables del estudio

\begin{tabular}{lccccc}
\hline \multicolumn{1}{c}{ Variable } & $\begin{array}{c}\text { Altos RISC-e } \\
(\mathrm{N}=57)\end{array}$ & $\begin{array}{c}\text { Bajos RISC-e } \\
(\mathrm{N}=62)\end{array}$ & $\mathrm{t}$ & $\mathrm{p}$ & $\mathrm{d}$ \\
\hline Metas Logro & $4.9(0.9)$ & $4.1(1.3)$ & 3.91 & 0.001 & 0.71 \\
Metas Rel Person. & $5.9(0.7)$ & $5.1(1)$ & 4.95 & 0.001 & 0.91 \\
Metas Afiliación & $4.0(1.6)$ & $3.1(1.5)$ & 3.19 & 0.002 & 0.58 \\
Metas IntrínsecasI & $8.3(0.5)$ & $7.7(0.8)$ & 4.07 & 0.001 & 0.69 \\
Metas Físico I & $7.3(0.8)$ & $6.9(1)$ & 2.32 & 0.02 & 0.42 \\
Autonomía & $5.6(0.8)$ & $5.0(0.9)$ & 3.41 & 0.001 & 0.63 \\
Vinculación & $5.8(0.5)$ & $5.2(0.7)$ & 4.62 & 0.001 & 0.85 \\
\hline
\end{tabular}

Fuente: elaboración propia 


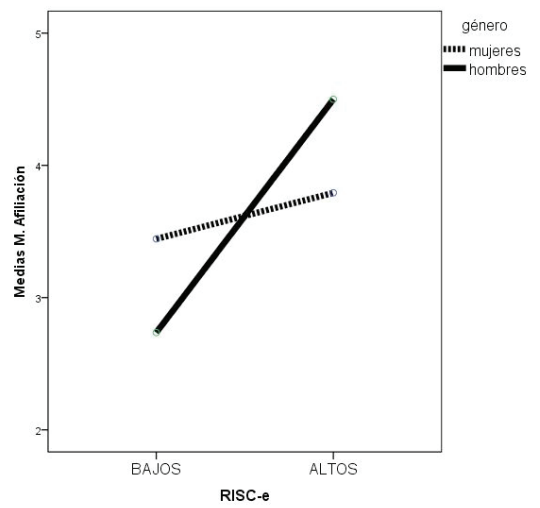

Figura 1. Interacción del Risc-e con la variable género en la predicción de las metas de afiliación.

Fuente: elaboración propia

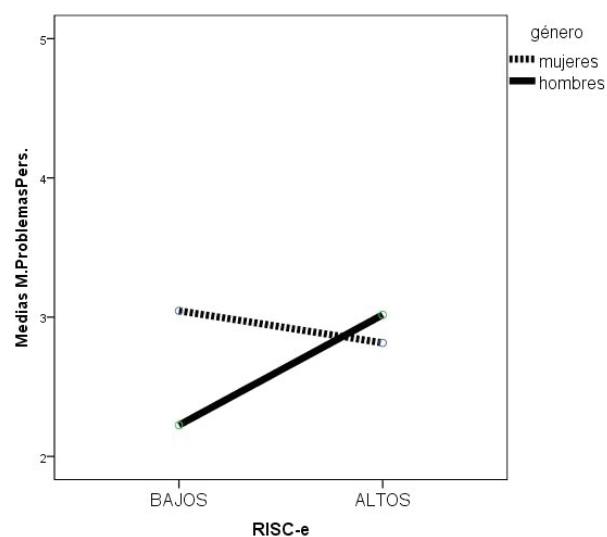

Figura 2. Interacción del Risc-e con la variable género en la predicción de las metas de problemas personales.

Fuente: elaboración propia

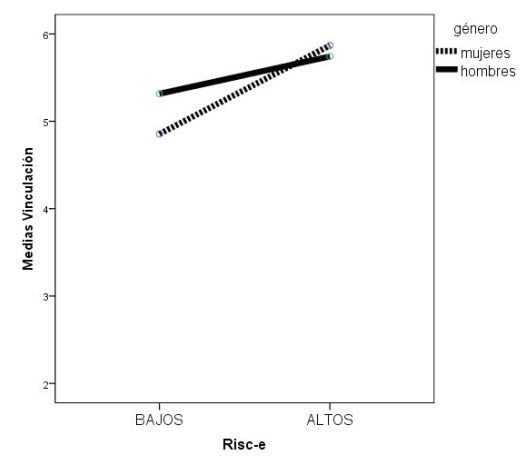

Figura 3. Interacción del Risc-e con la variable género en la predicción de la satisfacción de la necesidad de vinculación. Fuente: elaboración propia en el RISC-e. Los hombres solo se diferencian (marginalmente) en su menor interés por las metas de afiliación con respecto a las mujeres, cuando ambos puntúan bajos en el RISC-e $(\hat{\sigma}=2.7$ y $q=3.4)$, $t_{(60)}=1.88, p=0.06$, y no cuando puntúan alto $(\hat{\sigma}$ $=4.5 \mathrm{y} q=3.7$ ).

En el caso de las metas de superación de problemas personales, como se muestra en la Figura 2, son nuevamente los estudiantes los que cambian su interés en función de su puntuación en el RISC-e. Los hombres se diferencian significativamente en su menor interés por este tipo de metas con respecto a las mujeres, cuando ambos puntúan bajos en el RISC-e $(\hat{\sigma}=2.2$ y $q=3.1), t_{(60)}=2.68, p<0.05$, no cuando puntúan alto $((\hat{0}=3 \mathrm{y} q=2.8)$.

Por último, como se muestra en la Figura 3, en el caso de la satisfacción de la necesidad de vinculación son las estudiantes las que se muestran diferentes, en función de su puntuación en el RISC-e. Así, las mujeres que puntúan bajo tienen una satisfacción significativamente menor de la vinculación que los hombres que también puntúan bajo $(q=5$ y $\hat{o}=5.4), t_{(60)}=1.95, p=0.5$. Por el contrario, se sienten solo marginalmente más satisfechas que los hombres cuando ambos puntúan altos en el RISC-e $(q=5.9$ y $\widehat{o}=5.6), t_{(60)}=1.95, p=0.8$.

Relaciones entre el RISC y la satisfacción de las necesidades psicológicas de autonomía, competencia y vinculación.

Por último, nos interesaba explorar el papel del RISC-e y la importancia y probabilidad concedida a unas u otras metas en la satisfacción de las necesidades psicológicas organísmicas (Deci \& Ryan, 2000). En la Tabla 4, se observan los resultados de los análisis de regresión que mostraron un patrón predictivo significativo.

Como se puede observar, es la combinación de la puntuación en el RISC-e y las metas intrínsecas la que mejor predice la satisfacción en las necesidades de autonomía, competencia y vinculación. En el caso de la autonomía, tanto la importancia concedida a estas metas como las expectativas de obtenerlas predicen el $10 \%$ de la satisfacción en dicha necesidad, a diferencia de lo que ocurre en 


\section{TABLA 4}

Resumen de los análisis de regresiones múltiples de la satisfacción de necesidades psicológicas, en función del Risc-e y las metas Intrínsecas.

\begin{tabular}{|c|c|c|c|c|}
\hline Variable & B & SE B & $\beta$ & R2 \\
\hline Vinculación & & & & $0.13 * *$ \\
\hline Risc-e & 0.26 & 0.04 & $0.31 * *$ & \\
\hline Me IntrínsecasP & 0.11 & 0.03 & $0.14^{*}$ & \\
\hline Me IntrínsecasI & 0.02 & 0.05 & 0.02 & \\
\hline Autonomíaa & & & & $0.10^{* *}$ \\
\hline Risc-e & 0.24 & 0.05 & $0.21 * *$ & \\
\hline Me IntrínsecasP & 0.13 & 0.05 & $0.13 *$ & \\
\hline Competenciab & & & & $0.09 * *$ \\
\hline Me IntrínsecasP & 0.25 & 0.05 & $0.27 * *$ & \\
\hline Risc-e & 0.15 & 0.05 & $0.15^{*}$ & \\
\hline Me IntrínsecasI & 0.08 & 0.06 & 0.18 & \\
\hline
\end{tabular}

las otras necesidades, donde el peso reside en la probabilidad de conseguir dichas metas y no en la importancia que se les concede. Aunque los porcentajes de varianza explicado no son muy grandes (entre el 9\% y el 13\%), son destacables los valores de los pesos de la variable RISC-e, especialmente en el caso de la vinculación $(\beta=0.31)$.

\section{Discusión}

En este trabajo, se ha explorado una medida del autoconcepto relacional y su influencia en la elección de metas vitales y en la satisfacción de necesidades psicológicas. Cuando se habla de autoconcepto relacional, se hace referencia a la construcción del autoconcepto a partir de la inclusión de las relaciones significativas con los otros (Cross et al., 2000; Singelis, 1994).

Para medir esta dimesión relacional-interdepediente se llevó a cabo una traducción del RISC (Cross et al., 2000) cuya estructura unidimensional solo se pudo confirmar cuando se eliminaron los dos ítems negativos que se incluían en la escala original. Con esta nueva versión que denominamos RISC-e, que presentó un buen índice de consistencia y un buen ajuste al modelo propuesto por Cross et al., se confirmaron las predicciones iniciales: las mujeres puntúan más alto que los hombres, existen diferencias entre altos y bajos en el RISC-e y esta medida tiene un peso significativo, a la hora de predecir la satisfacción de las necesidades psicológicas. En este grupo de predicciones, también se ha confirmado -como lo había hecho Cross- la ausencia de una relación directa entre la puntuación de la escala y el bienestar medido por las emociones positivas y negativas. Además de confirmar estas predicciones ajustadas a los trabajos previos de Cross et al., se encontraron algunas relaciones interesantes que se comentarán con más detenimiento.

Por un lado, se encontró que la variable RISC tiene un papel modulador en las diferencias de género asociadas a la satisfacción de la necesidad de vinculación y a la importancia dada a las metas de afiliación y a las metas de superación de problemas personales. Así, la interacción entre los grupos del RISC-e y la variable género indica que las mujeres viven de una forma más intensa la construcción del autoconcepto relacional y la satisfacción de la necesidad de vinculación. Las mujeres que puntúan bajo en el RISC-e tienen una insatisfacción mayor que los hombres en la necesidad de vinculación y cuando puntúan en el grupo alto, una mayor satisfacción de dicha necesidad. Sin embargo, los datos más interesantes son los que igualan a hombres y mujeres en la importancia concedida a las metas de 
afiliación y de superación de problemas personales. Cuando los hombres puntúan alto en el RISC-e le dan la misma importancia a esas metas que las mujeres, cuando puntúan bajo ocurre algo distinto: le dan menos importancia.

Estos datos coinciden con la teoría de las expectativas de rol de género, de tal forma que hombres y mujeres pueden mostrar el mismo interés por las metas interpersonales, pero en la vida cotidiana se diferencian de forma significativa, de acuerdo a la normativa de rol de género que en cada momento marca la cultura (Barberá, 1998; Horgan \& Smith, 2006).

Con respecto a la diferencia entre estudiantes que puntúan alto y bajo en el RISC-e, es importante destacar que las diferencias se centran en las metas interpersonales, en las dos formas en que se han medido: a través del MOPI (metas de logro, relaciones personales y afiliación) y a través de la escala de Aspiraciones Vitales. En esta última, las diferencias más acusadas están en las metas intrínsecas y en el polo físico de la dimensión yo trascedente-yo físico. La importancia de estas últimas en la vida de los estudiantes ha sido constatada en estudios previos con sujetos españoles, en donde la salud física y psicológica predecían el 46\% y 54\% de la variaciones en la satisfacción autopercibida (Castro \& Sánchez, 2000). Tal como se encuentra aquí, los estudiantes con un autoconcepto relacional alto dan mayor importancia a las metas relacionadas con mantener relaciones con los otros, formar parte de una comunidad, conocerse a sí mismos y tener buena salud física.

En relación con la satisfacción de necesidades psicológicas, y en el sentido esperado, los estudiantes del grupo alto en el RISC muestran mayor satisfacción de su necesidad de vinculación que aquellos que puntúan bajo; lo mismo ocurre con la necesidad de autonomía. Esta relación entre vinculación y autonomía ha sido puesta a prueba en varios trabajos (Gore \& Cross, 2006; Gore et al., 2009), integrando el concepto de autoconcepto relacional interdependiente con el modelo de autoconcordancia de Sheldon y Elliot (1998), para explicar la elección de metas. Según este modelo, las personas eligen diferentes metas debido a dos razones fundamentales: por un lado, eligen metas que son coherentes con sus valores e intereses, es decir, por una motivación de autonomía, y eligen otras metas porque lo demanda la situación u otras personas cercanas. En este último caso, se trataría de motivos de control. En el primer caso se habla de metas autoconcordantes/congruentes (self-concordant), relacionadas con un mayor esfuerzo, bienestar y ajuste psicológico (Sheldon \& Elliot, 1998, 1999; Sheldon $\&$ Hauser-Marko, 2001). Para Cross y Gore, estas metas auto-concordantes son un ejemplo de metas motivadas por razones personales-autónomas (PARs) en contraste con las metas motivadas por razones relacionales-autónomas (RARs), es decir, autónomas porque cada cual las elige para expresar sus deseos y valores, pero con la particularidad de que expresan necesidades, deseos y compromisos con los otros. Son estas últimas metas las que se relacionan más directamente con el esfuerzo y la persistencia, aunque ambas aparecen directamente asociadas al bienestar y las emociones positivas (Gore \& Cross, 2006).

El último objetivo del presente estudio fue explorar la capacidad de esta variable, de diferencias individuales, medida por el RISC-e, para predecir la satisfacción de las necesidades psicológicas de autonomía, competencia y vinculación. Los resultados muestran unos porcentajes de varianza pequeños pero significativos. Cuando se predice la satisfacción de las tres necesidades psicológicas se hace explícito el peso de esta variable en combinación con la probabilidad de conseguir las metas intrínsecas, para el caso de la satisfacción de la vinculación y de la competencia. Para el caso de la satisfacción de la autonomía, lo hace en combinación con la importancia dada a dichas metas.

Este estudio tiene algunas limitaciones que obligan a tomar con precaución sus resultados. Aunque los índices obtenidos, tanto en el AFE como en el AFC son buenos y permiten hacer predicciones en la muestra utilizada, se requeriría de una muestra mayor para poder validar definitivamente la versión en castellano del RISC. Por otro lado, solo se han incluido estudiantes de grado o de la licenciatura en Psicología. Aunque son de distintas promociones, sería necesario ampliar la muestra a estudiantes 
de distintas carreras, especialmente porque los de psicología podrían tener una sensibilidad mayor a los contenidos interpersonales.

Más en general, aunque las limitaciones de este estudio son las propias de los trabajos correlacionales, los autores consideran que los datos aportan evidencias de la utilidad de esta medida de diferencias individuales que se mide con el RISC-e como variable que se debe tener en cuenta a la hora de explicar la motivación de los estudiantes en la elección de sus metas vitales y la satisfacción de sus necesidades psicológicas.

\section{Referencias}

Barberá, E. (1998). Psicología del género. Barcelona: Ariel. Bentler, P. M. (1989). Comparative fit indexes in structural models. Psychological Bulletin, 107(2), 238246.

Breines, J. G., \& Chen, S. (2013). Activating the inner caregiver: The role of support-giving schemas in increasing state self-compassion. Journal of Experimental Social Psychology, 49(1), 58-64.

Byrne, B. M. (2010). Structural equation modeling with AMOS. New York: Routledge Taylor \& Francis.

Carver, C. S. (2006). Approach, avoidance, and the self-regulation of affect and action. Motivation and Emotion, 30(2), 105-110.

Castro, A., \& Sánchez, M. P. (2000). Objetivos de vida y satisfacción autopercibida en estudiantes universitarios. Psicothema, 12(1), 87-92.

Cross, S. E., Bacon, P., \& Morris, M. (2000). The relational-interdependent self-construal and relationships. Journal of Personality and Social Psychology, 78(4), 791-808.

Cross, S. E., Hardin, E., \& Gercek-Swing, B. (2009). Independent, relational and collective-interdependent self-construals. En M. R. Leary \& R. H. Hoyle (Eds.), Handbook of individual differences in social behavior (pp. 512-526). New York: Guilford Press.

Cross, S. E., Hardin, E., \& Gercek-Swing, B. (2011). The what, how, why, and where of self-construal. Personality and Social Psychology Review, 15(2), 142-179.

Cross, S. E., Morris, M., \& Gore, J. (2002). Thinking about oneself and others: The relational-inter- dependent self-construal and social cognition. Journal of Personality and Social Psychology, 82(3), 399-418.

Deci, E. L., \& Ryan, R. M. (2000). The 'what' and 'why' of goal pursuits: Human needs and the selfdetermination of behavior. Psychological Inquiry, 11(4), 227-268.

Deci, E. L., \& Ryan, R. M. (2002). Handbook of selfdetermination research. Rochester: University of Rochester Press.

Gámez, E., \& Marrero, H. (2001). Metas y motivos en la elección de la carrera de psicología. Revista Española de Motivación y Emoción, 2, 9-22.

Gámez, E., \& Marrero, H. (2003). Metas y motivos en la elección de la carrera universitaria: un estudio comparativo entre psicología, derecho y biología. Anales de Psicología, 19(1), 121-131.

Gore, J. S., \& Cross, S. E. (2006). Pursuing goals for us: Relationally-autonomous reasons in long-term goal pursuit. Journal of Personality and Social Psychology, 90(5), 858-861.

Gore, J. S., Cross, S. E., \& Kanagawa, C. (2009). Acting in our interests: Relational self-construal and goal motivation across cultures. Motivation and Emotion, 33(1), 75-87.

Grouzet, F. M., Kasser, T., Ahuvia, A., Dols, J. M. F., Kim, Y., Lau, S., \& Sheldon, K. M. (2005). The structure of goals across 15 cultures. Journal of Personality and Social Psychology, 89(5), 800-816.

Hardin, E. E. (2006). Convergent evidence for the multidimensionality of self-construal. Journal of Cross-cultural psychology, 37(5), 516-521.

Horgan, T. G., \& Smith, J. L. (2006). Interpersonal reasons for interpersonal perceptions: Genderincongruent purpose goals and nonverbal judgment accuracy. Journal of Nonverbal Behaviour, 30(3), 127-140.

Jöreskog, K. G., \& Sörbom, D. (1993). LISREL 8: Structural equation modeling with the SIMPLIS command language: Chicago: Scientific Software International.

Kasser, T. (2002). Sketches for a self-determination theory of values. En E. L. Deci \& R. M. Ryan (Eds.), Handbook of self-determination research (pp. 123140). Rochester, NY: University of Rochester Press. 


\section{Elena Gámez Armas, Jose Miguel Díaz Gómez, Hipólito Marrero Hernández, Ma Paz Galindo Galindo, Alicia Breva Asensio}

Kasser, T., \& Ryan, R. M. (1996). Further examining the American dream: Differential correlates of intrinsic and extrinsic goals. Personality and Social Psychology Bulletin, 22(3), 280-287.

Kitayama, S. (2002). Culture and basic psychological processes-Toward a system view of culture: Comment on Oyserman et al. (2002). Psychological Bulletin, 128(1), 89-96.

Markus, H. R., \& Kitayama, S. (1991). Culture and the self: Implications for cognition, emotion, and motivation. Psychological Review, 98(2), 224-253.

Mattingly, B. A., Oswald, D. L., \& Clark, E. M. (2011). An examination of relational-interdependent selfconstrual, communal strength, and pro-relationship behaviors in friendships. Personality and Individual Differences, 50(8), 1243-1248.

Muñiz, J., \& Hambleton, R. K. (1996). Directrices para la traducción y adaptación de los tests. Papeles del Psicólogo, 66, 63-70.

Nisbett, R. E. (2003). The geography of thought. New York: Free Press.

Sheldon, K. M. (2005). Positive value change during college: Normative trends and individual differences. Journal of Research in Personality, 39(2), 209-223.

Sheldon, K. M., \& Elliot, A. J. (1998). Not all personal goals are personal: Comparing autonomous and controlled reasons as predictors of effort and attainment. Personality and Social Psychology Bulletin, 24(5), 546-557.

Sheldon, K. M., \& Elliot, A. J. (1999). Goal striving, need-satisfaction, and longitudinal well-being: The self-concordance model. Journal of Personality and Social Psychology, 76(3), 482-497.

Sheldon, K. M., Gunz, A., Nichols, C., \& Ferguson, Y. (2010). Extrinsic value orientation and affective forecasting: Over-estimating the rewards, underestimating the costs. Journal of Personality, 78(1), $149-178$
Sheldon, K. M., \& Houser-Marko, L. (2001). Self-concordance, goal-attainment, and the pursuit of happiness: Can there be an upward spiral? Journal of Personality and Social Psychology, 80(1), 152-165.

Sheldon, K. M., \& Kasser, T. (2008). Psychological threat and goal striving. Motivation and Emotion, 32(1), 37-45.

Sheldon, K. M., Ryan, R., Deci, E., \& Kasser, T. (2004). The independent effects of goal contents and motives on well-being: It's both what you pursue and why you pursue it. Personality and Social Psychology Bulletin, 30(4), 475-486.

Singelis, T. M. (1994).The measurement of independient and interdependient self-construals. Personality and Social Psychology Bulletin, 20(5), 580-591.

Singelis, T. M., Bond, M. H., Lai, S. Y., \& Sharkey, W. F. (1999). Unpackaging culture's influence on self-esteem and embarrassability: The role of selfconstruals. Journal of Cross-Cultural Psychology, 30(3), 315-341.

Singelis, T. M., \& Brown, W. J. (1995). Culture, self, and collectivist communication: Linking culture to individual behavior. Human Communication Research, 21(3), 354-389.

Triandis, H. C. (1994). Culture and social behavior. New York: MacGraw-Hill.

Vansteenkiste, M., Soenens, B., \& Duriez, B. (2008). Presenting a positive alternative to materialistic strivings and the thin-ideal: Understanding the effects of extrinsic relative to intrinsic goal pursuits. En S. J. Lopez (Ed.), Positive psychology: exploring the best in people (pp. 57-86). Westport, CT: Greenwood Publishing Company.

Watson, D., Clark, L. A., \& Tellegen, A. (1988). Development and validation of brief measures of positive and negative affect: The PANAS scales. Journal of Personality and Social Psychology, 54(6), 1063-1070. 


\section{Apéndice}

Traducción del RISC al español (RISC-e)

Por favor, lee cada una de las frases que te presentamos y valora (marcar con una $\mathrm{X}$ ) en qué medida se relacionan con tu vida, es decir, en qué medida te identificas con la afirmación que expresa cada frase.

Utiliza los valores de la escala que te presentamos

$$
1 \ldots \ldots .2 \ldots . .3 \ldots . . .4 \ldots \ldots . . \ldots \ldots 6 \ldots . . .7
$$

Totalmente Moderadamente Totalmente en desacuerdo de acuerdo de acuerdo

Mis relaciones son un importante reflejo de quien soy.
Cuando me siento muy cerca de alguien, suelo sentir como si esa persona representara una parte importante de quien soy.

Suelo sentir un fuerte sentimiento de orgullo cuando alguien cercano a mí consigue un logro importante.

Pienso que uno de los aspectos más importantes de quien soy yo puede apreciarse a través de mis amigos íntimos y como son.

Cuando pienso en mí, también suelo pensar en mi familia y mis amigos íntimos.

1234567

Si alguien hiere a alguien cercano a mí yo también me siento herida/o.

1234567

En general, mis relaciones íntimas son una parte importante de mi propia imagen.

1234567

En general, mis relaciones íntimas tienen muy poco que ver con cómo me siento.

1234567

Mis relaciones íntimas son poco importantes en relación con qué tipo de persona soy.

1234567

Mi sentimiento de orgullo está relacionado con quienes son mis amigos íntimos.

1234567

Cuando establezco una relación íntima con alguien, normalmente surge un fuerte sentimiento de identificación con esa persona. 
